\title{
Recycling of Waste Rubber by the Manufacturing of Foamed Polyurethane-Based Composites-Current State and Perspectives ${ }^{\dagger}$
}

\author{
Aleksander Hejna *, Krzysztof Formela, Łukasz Zedler, Paulina Kosmela and Adam Olszewski \\ Department of Polymer Technology, Gdańsk University of Technology, Narutowicza 11/12, 80-233 Gdańsk, \\ Poland; krzform1@pg.edu.pl (K.F.); lukasz.zedler@pg.edu.pl (Ł.Z.); paulina.kosmela@pg.edu.pl (P.K.); \\ adam.olszewski@student.pg.edu.pl (A.O.) \\ * Correspondence: aleksander.hejna@pg.gda.pl; Tel.: +48-58-347-2234 \\ + Presented at the First International Conference on “Green" Polymer Materials 2020, 5-25 November 2020; \\ Available online: https://cgpm2020.sciforum.net/.
}

Published: 4 November 2020

\begin{abstract}
Worn car tires are disruptive waste, and the issue of their management is crucial for the natural environment. In many countries, the primary method of end-of-life tires utilization is energy recovery. However, more effective and beneficial for the environment is material recycling. Using them for the production of polymer-rubber composites seems to be an auspicious direction of research. Incorporation of ground tire rubber into polyurethane matrix should be considered as a method of waste rubber utilization. Moreover, it could significantly reduce the use of petroleumbased polyols and isocyanates, which are commonly considered as toxic chemicals. Therefore, the total impact on the environment could be noticeably reduced, which should be considered as very beneficial step towards more "green" polymer composites. This work aims to summarize the literature reports related to the foamed polyurethane/ground tire rubber composites. It particularly emphasizes the need for compatibilization of these materials by the enhancement of interfacial interactions between the polyurethane matrix and rubber filler phase, which significantly affect the performance properties of prepared materials. As an example, we presented our research results. Besides, future trends and limitations related to this type of composite materials are underlined.
\end{abstract}

Keywords: recycling; ground tire rubber; polyurethane foams; composites; rubber modification; matrix-filler interactions

\section{Introduction}

The increase in the number of cars in the European Union countries, including Poland, results in introducing more car tires to the market. In 2018, more than 5.1 million tons of car tires were produced in Europe, and in general, more than 465 million tires were introduced to the European market [1]. These data indicate an upward trend stable over the last decade. The dominance of imports over exports is also increasing. Forecasts indicating an increase in the number of cars suggest that the number of car tires in Europe will continue to grow in the coming years. Tires are a typical operating element of a car, so it is necessary to replace them regularly, which in the case of relatively economical driving takes place on average every three years. As a result, in Europe, almost 3.3 million tons of car tires are being withdrawn from use annually [1]. Due to their quantity and durability (their time of degradation in the natural environment is estimated at over 100 years), the used car tires are a nuisance and should be used industrially. Due to global problems with waste tires, recycling has been a prevalent research topic for many years. Due to their chemical composition and cross-linked structure, tires' recycling is much more complicated than the recycling of thermoplastics, which are the raw waste from the packaging industry. Used tires are divided into three main groups: partially 
used tires (they have an even more significant than minimum tread depth), used retread tires, and used tires. The last two groups should be recycled. Storage of used car tires is currently prohibited, so their recovery and recycling are mandatory. Tire recycling can be divided into two types due to the technology's nature: material recycling and energy recovery (from which sometimes is also distinguished pyrolysis as chemical recycling). Material recycling is the most preferred form and consists of converting the tires into products with a reasonable value, such as ground tire rubber (GTR). Appropriate selection of composition and the method and parameters of processing allows obtaining secondary materials with high efficiency and good quality products. Energy recovery consists of burning rubber waste and partial recovery of energy used to produce it.

\section{Ground Tire Rubber in Plastics Technology}

Currently, the products of material recycling of car tires are used to fill in the construction of tunnels, road surface layers, sports surfaces, and the production of car rugs, wipers, cattle mats, or as asphalt modifiers [2]. However, these applications still do not guarantee sufficient demand for processed rubber waste. Therefore, much research is currently being conducted on searching for potential applications for GTR, among others, in the plastics industry [3]. Composite materials' production seems to be the most promising research direction due to the multitude of polymer rubber products' potential applications. So far, ground tire rubber has been used as a filler in composites based on polyurethanes, polyolefins, or rubber [4,5]. However, the authors point to the main problem limiting the broader use of rubber waste in producing polymeric composites - low compatibility of GTR with polymer matrices [6]. As a result, the mechanical properties of the obtained composites are insufficient for potential applications. The solution to this problem is the modification of rubber waste, consisting, e.g., in the oxidation of the rubber surface, partial devulcanization of the GTR, or grafting of various chemical compounds on its surface [7]. This type of modification allows us to give rubber waste new functional characteristics. In the literature, many GTR modification methods have been described, including mechanical, thermomechanical, mechanical-chemical, and other methods, such as microwave, ultrasound, microorganisms, or $\mathrm{CO}_{2}$ in the supercritical state [8]. Nevertheless, the vast majority of the described methods are based on periodical processes. As a result, the presented methods have several drawbacks, among which it is necessary to mention:

- The use of organic and inorganic solvents-therefore, the product usually requires additional treatment, which complicates the process (additional unit operations), generates significant amounts of wastewater, and extends the processing time. Moreover, for many years it has been postulated that the technology should use as few solvents as possible, if not necessary [9].

- The need to start and stop production daily, and sometimes more often, reduces the efficiency of the process and its economic aspect.

- The use of microwave and plasma reactors is costly and does not provide too high process efficiency [9].

These disadvantages effectively limit the industrial application of the above-mentioned periodical methods. Therefore, it is reasonable to look for methods of modification of ground tire rubber of continuous character. A potentially very effective method is the modification of fillers in situ in the process of reactive extrusion. This process is fast, relatively cheap, and considered a proecological alternative to traditional, periodical modification [10]. The use of reactive extrusion allows for an exact adjustment of the modification conditions by changing the process parameters such as temperature in individual zones of the cylinder, screw speed, capacity, material residence time in the extruder, and finally, the size of shear forces acting on the material regulated by changing the screw configuration. This process's advantage is the absence of solvents (although this method does not exclude their use), which eliminates additional cleaning processes. These factors cause constant development and increased interest in this method. Even though reactive extrusion is a process known for many years, its use to modify GTR is a relatively new research direction. 


\section{Ground Tire Rubber in Polyurethane Foams}

As mentioned above, adequately modified rubber had a structure that ensures sufficiently strong interactions at the matrix-filler phase boundary and could be successfully used to produce foamed polyurethane rubber composites. Polyurethane foams are an extensive group of materials, which can be divided into rigid and flexible foams most simply. The first group is mainly used as thermal insulation materials. The most important in their case is to obtain an appropriate cellular structure, which will provide the lowest possible value of the thermal conductivity coefficient. The introduction of ground tire rubber into rigid polyurethane foams could positively affect the final product's thermal insulation properties due to the lower value of the thermal conductivity coefficient of rubber compared to solid polyurethane $(\sim 160$ and $\sim 220 \mathrm{~mW} /(\mathrm{m} \cdot \mathrm{K}))$ [11]. Nevertheless, to achieve this effect, it would be necessary to adjust the cellular structure accordingly, and so far, there are no such reports in the literature.

Research works related to incorporating ground tire rubber into rigid polyurethane foams focused mainly on the mechanical and thermal performance of composite materials. In our previous works [12,13], we introduced GTR into fully petrochemical and crude glycerol-based rigid polyurethane foams. To enhance the composites' compatibility, thermo-mechanical treatment of ground tire rubber in the extruder was applied. Incorporation of solid rubber particles resulted in the polyol mixtures' increased viscosity, implicating a higher apparent density of foams. This phenomenon was more pronounced for materials filled with pretreated GTR, which, together with the lower temperatures recorded during polymerization, points to the enhanced interactions with isocyanates. Maximum temperatures were decreased from almost $110^{\circ} \mathrm{C}$ for neat polyurethane foam to $80-85^{\circ} \mathrm{C}$ for the material filled with 30 parts by weight of untreated GTR, and to $70-75{ }^{\circ} \mathrm{C}$ when pretreated GTR was applied.

Nevertheless, despite enhanced interactions between rubber particles and the polyurethane system, microscopic analysis revealed the cellular structure's disruption, which increased the average cell size and content of open cells. It also caused the rise of apparent density. Such effects are unfavorable for the potential applications of this type of material as thermal insulation. A higher apparent density of foam implicates an increased share of solid material in a given volume [14]. As mentioned above, values of thermal conductivity coefficient of rubber and solid polyurethane are $\sim 160$ and $\sim 220 \mathrm{~mW} /(\mathrm{m} \cdot \mathrm{K})$, while for $\mathrm{CO}_{2}$ and air, it is 15.3 and $24.9 \mathrm{~mW} /(\mathrm{m} \cdot \mathrm{K})$, respectively. Incorporating the physical blowing agents may be even more beneficial since they are often characterized by the thermal conductivity at the level $\sim 10 \mathrm{~mW} /(\mathrm{m} \cdot \mathrm{K})$ [15]. Another parameter affecting insulation properties is the average cell size. It is correlated with heat radiation. It has been proved that the increase in average cell diameter from 0.25 to $0.60 \mathrm{~mm}$ caused an increase in the thermal conductivity coefficient by almost $50 \%$ [15]. The content of open cells in foam also affects the insulation properties since its higher values facilitate heat convection through cellular materials. Therefore, obtained results strongly point to the need for proper adjustment of foams' formulations to maintain all cellular structure parameters when solid GTR particles are introduced. It was lately confirmed by Silva et al. [16], who introduced from 5 to $20 \mathrm{wt} \%$ of ground tire rubber into polyurethane foam formulation. They noted the decrease of average cell size with the increasing GTR content attributed to the nucleation effect of solid particles. Nevertheless, at the same time, the cellular structure became significantly less homogeneous, with thinner cell walls and a noticeably higher amount of broken, open cells. As a result, despite the increase of the apparent density from 36.5 to even $48.7 \mathrm{~kg} / \mathrm{m}^{3}$, compressive and flexural strength was reduced from the initial $259 \mathrm{kPa}$ and $1.96 \mathrm{MPa}$ to $181 \mathrm{kPa}$ and $0.91 \mathrm{MPa}$.

In our further work [17], we adjusted foams' formulations and introduced ground tire rubber with a smaller particle size. Such changes resulted in a similar cellular structure of neat polyurethane foam and foam containing 20 parts by weight of GTR. The average cell size for these materials was around $200 \mu \mathrm{m}$, which is a beneficial value for the insulation properties. Moreover, the low content of broken, open cells was noted. As a result, noticeable enhancement of the mechanical performance was observed, compressive strength was increased by 59 and $45 \%$, parallel and perpendicular to the 
foam rise, respectively. Simultaneously, the observed cellular structure of composite foams should also be very beneficial for their insulation properties.

Flexible polyurethane foams seem to be more promising in terms of the use of ground tire rubber, mainly due to their multitude of applications. These materials are commonly used in furniture, automotive, construction, or packaging industries. They are applied in products where the material cost is often an essential aspect, so the use of relatively cheap GTR could reduce the cost of the material and significantly increase its attractiveness to potential buyers. Literature data indicate that the introduction of ground tire rubber into flexible polyurethane foams has a positive effect on improving damping properties both in the direction of mechanical and acoustic vibrations [18].

Cachaço et al. [19] observed a significant increase in the compressive strength and modulus of elasticity as a result of introducing ground tire rubber into the foamed, flexible polyurethane matrix, Gayathri et al. [18] noted an increase in the tensile and compressive strength of foams with the addition of GTR. Moreover, they indicate a significant increase in the material's sound absorption coefficient, which is a great advantage of using foams as damping materials. A similar effect related to sound absorption was observed by Zhang et al. [20]. Moreover, they studied the influence of the addition of unmodified and partially devulcanized rubber on the material's structure and properties. They observed that the devulcanization of GTR allowed reducing the foam's cell size, which should be considered beneficial in terms of mechanical properties. Besides, the introduction of ground tire rubber into the foam allowed increasing the loss modulus, resulting in the enhanced ability to dissipate the energy.

In our work related to the flexible polyurethane foams filled with GTR [21], we also applied neat and thermo-mechanically treated filler. Independently of the type of GTR, similar values of apparent density were obtained. For the neat polyurethane matrix, it was $290 \mathrm{~kg} / \mathrm{m}^{3}$, while the incorporation of 10 and 30 parts by weight of fillers caused its rise to $299-316 \mathrm{~kg} / \mathrm{m}^{3}$. For unmodified ground tire rubber, the increase of apparent density was followed by enhancing compressive strength, but this effect was not noted for pretreated GTR. It was associated with the enhanced interfacial interactions with the polyurethane system, especially with isocyanates. Because part of isocyanate reacted with rubber particles instead of polyols, the cellular structure was not adequately developed. The SEM analysis confirmed such an assumption. This work confirmed the need to adjust foams' formulations when GTR, especially previously modified, is introduced as a filler.

As mentioned above, foamed polyurethane-rubber composites could be used in furniture, automotive, construction, or packaging industry to replace the unfilled polyurethane foams. Apart from the packaging industry, these branches of industry require the material to meet the relevant standards related to flammability and smoke-generation, and the criteria for allowing the material to be used are getting stricter and stricter. The material's properties such as flammability, the time and speed of burning, the amount and speed of generated heat, the amount and toxicity of the fumes emitted are essential from the fire safety point of view. Unmodified polyurethanes are flammable materials, and during their degradation, carbon and nitrogen oxides or hydrogen cyanide, which are harmful to health, may be generated [22]. The use of ground tire rubber as a filler is not expected to improve the fire's material resistance. Nevertheless, it is essential to investigate this aspect of polyurethane/GTR composites, and so far, hardly any works have included this issue. Ryszkowska et al. [23] investigated the flammability and flame retardancy of semi-rigid foams filled with 25 parts by weight of ground tire rubber modified additionally by the expanded graphite, organophosphorus compound Fyrol PNX and their combination. Applied modifications allowed to increase the oxygen index by more than $30 \%$ and reduce the material's flammability determined by the cone calorimeter tests. Flame retarded foams were characterized by a significantly reduced total heat released (even by more than $65 \%$ ) and heat release rate (even by more than $71 \%$ ) compared to unmodified composite. The results of this work show a very high potential of polyurethane-rubber foamed composites. However, it is necessary to identify further the topic of reducing this type of composites' flammability.

An exciting possibility related to the pretreatment of ground tire rubber before the manufacturing of foamed polyurethane composites is incorporating flame retardants into rubber 
modification processes. They could be chemically bonded, deposited on rubber particles' surface, or even used to swell them like oils. The multitude of possibilities is related to the wide range of commercially available flame retardants. An essential aspect of their use is that, apart from lowering the flammability, they may also affect other polyurethane material properties, e.g., mechanical strength. Examples include liquid organophosphorus compounds or solid aluminosilicates. The first group of compounds is also used as plasticizers of various plastics, so their introduction into polyurethane will cause partial plasticization of the matrix, influencing processing and mechanical strength. Simultaneously, they may also swell rubber leading to the enhanced interfacial interactions with the polyurethane matrix. On the other hand, solid flame retardants, such as aluminosilicates, will cause stiffening of the material. Therefore, it is crucial for the material's properties to choose the right flame retardant for a specific material or composite, considering its final application.

\section{Conclusions and Future Trends}

The incorporation of ground tire rubber into a foamed polyurethane matrix is an exciting issue due to the wide range of properties and applications of polyurethane foams. Nevertheless, as shown based on literature reports, independently of the selected type of foamed polyurethane matrix, it is essential to provide a sufficient level of interfacial interactions with the simultaneous maintaining of the proper cellular structure. It could be achieved by the adjustment of foams' formulations and proper modifications of ground tire rubber.

In the first case, it is crucial to properly select the catalysts and surfactants essential for the generation of foams' cellular structure. Simultaneously, the polyol mixture's viscosity should be taken into account since it is noticeably affected by the introduction of GTR particles. Finally, depending on the level of rubber surface development, GTR would interact or even chemically react with isocyanates, so the corrections related to their amounts should be made.

Considering modifications of ground tire rubber, it is crucial to provide the desired level of chemical reactions and physical interactions between GTR particles and in situ formed polyurethane matrix. As mentioned before, pretreatment of ground tire rubber can be performed to modify its surface and reduce the average particle size or introduce additional modifiers such as flame retardants. Another issue that has to be considered during future research is the repeatability of the modification processes and storage stability of obtained products. To overcome these issues, continuous modification methods, such as the reactive extrusion, should be investigated.

Having in mind all the issues mentioned in the presented work, we are currently running the research project, whose goal is to develop the continuous method of ground tire rubber modification, aimed at enhancing the interfacial interactions between GTR and foamed polyurethane matrix. Modified rubber particles would be introduced into polyurethanes during composites' manufacturing with reduced flammability, which could be applied as vibration or sound damping materials.

Author Contributions: A.H., K.F. and Ł.Z. analyzed the literature data; A.H., P.K. and A.O. wrote the paper. All authors have read and agreed to the published version of the manuscript.

Acknowledgments: This work was supported by The National Centre for Research and Development (NCBR, Poland) in the frame of LIDER/3/0013/L-10/18/NCBR/2019 project-Development of technology for the manufacturing of foamed polyurethane-rubber composites for the use as damping materials.

Conflicts of Interest: The authors declare no conflict of interest. 


\section{References}

1. European Tyre \& Rubber Manufacturers' Association, European Tyre \& Rubber Industry Statistics Edition 2019. Available online: http://www. https://www.etrma.org/wp-content/uploads/2019/10/20200326Statistics-booklet-2019-Final-for-web-upload.pdf (accessed on 15 October 2020).

2. Thomas, B.S.; Gupta, R.C. A comprehensive review on the applications of waste tire rubber in cement concrete. Renew. Sust. Energy Rev. 2016, 54, 1323-13335, doi:10.1016/j.rser.2015.10.092.

3. Ramarad, S.; Khalid, M.; Ratnam, C.T.; Luqman Chuah, A.; Rashmi, W. Waste tire rubber in polymer blends: A review on the evolution, properties and future. Prog. Mater. Sci. 2015, 72, 100-140, doi:10.1016/j.pmatsci.2015.02.004.

4. Danielli Bastos de Sousa, F.; Scuracchio, C.H.; Hu, G.H.; Hoppe, S. Effects of processing parameters on the properties of microwave-devulcanized ground tire rubber/polyethylene dynamically revulcanized blends. J. Appl. Polym. Sci. 2016, 133, 43503, doi:10.1002/app.43503.

5. Aoudia, K.; Azem, S.; Hocine, N.A.; Gratton, M.; Pettarin, V.; Seghar, S. Recycling of waste tire rubber: Microwave devulcanization and incorporation in a thermoset resin. Waste Manag. 2017, 60, 471-481, doi:10.1016/j.wasman.2016.10.051.

6. Hernández, E.H.; Gámez, J.F.H.; Cepeda, L.F.; Muñoz, E.J.C.; Corral, F.S.; Rosales, S.G.S.; Velázquez, G.N.; Morones, P.G.; Martínez, D.I.S. Sulfuric acid treatment of ground tire rubber and its effect on the mechanical and thermal properties of polypropylene composites. J. Appl. Polym. Sci. 2017, 134, 44864, doi:10.1002/app.44864.

7. Cheng, X.W.; Long, D.; Huang, S.; Li, Z.Y.; Guo, X.Y. Time effectiveness of the low-temperature plasma surface modification of ground tire rubber powder. J. Adhes. Sci. Technol. 2015, 29, 1330-1340, doi:10.1080/01694243.2015.1026958.

8. Colom, X.; Faliq, A.; Formela, K.; Canavate, J. FTIR spectroscopic and thermogravimetric characterization of ground tyre rubber devulcanized by microwave treatment. Polym. Test. 2016, 52, 200-208, doi:10.1016/j.polymertesting.2016.04.020.

9. Rungrodnimitchai, S.; Kotatha, D. Chemically modified ground tire rubber as fluoride ions adsorbents. Chem. Eng. J. 2015, 282, 161-169, doi:10.1016/j.cej.2015.03.038.

10. Formela, K.; Klein, M.; Colom, X.; Saeb, M.R. Investigating the combined impact of plasticizer and shear force on the efficiency of low temperature reclaiming of ground tire rubber (GTR). Polym. Degrad. Stabil. 2016, 125, 1-11, doi:10.1016/j.polymdegradstab.2015.12.022.

11. Hejna, A.; Kirpluks, M.; Kosmela, P.; Cabulis, U.; Haponiuk, J.; Piszczyk, Ł. The influence of crude glycerol and castor oil-based polyol on the structure and performance of rigid polyurethane-polyisocyanurate foams. Ind. Crop. Prod. 2017, 95, 113-125, doi:10.1016/j.indcrop.2016.10.023.

12. Piszczyk, Ł.; Hejna, A.; Formela, K.; Danowska, M.; Strankowski, M. Rigid polyurethane foams modified with ground tire rubber-Mechanical, morphological and thermal studies. Cell. Polym. 2015, 2, 45-62, doi:10.1177\%2F026248931503400201.

13. Piszczyk, Ł.; Hejna, A.; Danowska, M.; Strankowski, M.; Formela, K. Polyurethane/ground tire rubber composite foams based on polyglycerol: Processing, mechanical and thermal properties. J. Reinf. Plast. Compos. 2015, 34, 708-717, doi:10.1177\%2F0731684415579089.

14. Hejna, A.; Kosmela, P.; Kirpluks, M.; Cabulis, U.; Klein, M.; Haponiuk, J.; Piszczyk, Ł. Structure, Mechanical, Thermal and Fire Behavior Assessments of Environmentally Friendly Crude Glycerol-Based Rigid Polyisocyanurate Foams. J. Polym. Environ. 2017, 26, 1854-1868, doi:10.1007/s10924-017-1086-2.

15. Randall, D.; Lee, S. The Polyurethanes Book; John Wiley \& Sons, Ltd.: New York, NY, USA, 2002.

16. Silva, N.G.S.; Cortat, L.I.C.O.; Orlando, D.; Mulinari, D.R. Evaluation of rubber powder waste as reinforcement of the polyurethane derived from castor oil. Waste Manag. 2020, 116, 131-139, doi:10.1016/j.wasman.2020.07.032.

17. Formela, K.; Hejna, A.; Zedler, Ł.; Przybysz, M.; Ryl, J.; Saeb, M.R.; Piszczyk, Ł. Structural, thermal and physico-mechanical properties of polyurethane/brewers' spent grain composite foams modified with ground tire rubber. Ind. Crop. Prod. 2017, 108, 844-852, doi:10.1016/j.indcrop.2017.07.047.

18. Gayathri, R.; Vasanthakumari, R.; Padmanabhan, C. Sound absorption, thermal and mechanical behavior of polyurethane foam modified with nano silica, nano clay and crumb rubber fillers. Int. J. Sci. Eng. Res. 2013, 4, 301-308.

19. Cachaço, A.G.; Afonso, M.D.; Pinto, M.L. New applications for foam composites of polyurethane and recycled rubber. J. Appl. Polym. Sci. 2013, 129, 2873-2881, doi:10.1002/app.38962. 
20. Zhang, X.; Lu, Z.; Tian, D.; Li, H.; Lu, C. Mechanochemical devulcanization of ground tire rubber and its application in acoustic absorbent polyurethane foamed composites. J. Appl. Polym. Sci. 2013, 127, 4006-4014, doi:10.1002/app.37721.

21. Piszczyk, Ł.; Hejna, A.; Formela, K.; Danowska, M.; Strankowski, M. Effect of ground tire rubber on structure, mechanical and thermal properties of flexible polyurethane foams. Iran. Polym. J. 2015, 24, 75-84, doi:10.1007/s13726-014-0301-4.

22. McKenna, S.T.; Hull, T.R. The fire toxicity of polyurethane foams. Fire Sci. Rev. 2016, 5, 3, doi:10.1186/s40038-016-0012-3.

23. Ryszkowska, J.; Leszczyńska, M.; Auguścik, M.; Bryśkiewicz, A.; Półka, M.; Kukfisz, B.; Wierzbicki, Ł.; Aleksandrowicz, J.; Szczepkowski, L.; Oliwa, R. Cores of composite structures made of semi-rigid foams for use as protecting shields for firefighters. Polimery 2018, 63, 125-133, doi:10.14314/polimery.2018.2.6.

Publisher's Note: MDPI stays neutral with regard to jurisdictional claims in published maps and institutional affiliations.

(C) 2020 by the authors. Submitted for possible open access publication under the terms and conditions of the Creative Commons Attribution (CC BY) license (http://creativecommons.org/licenses/by/4.0/). 